\title{
La tumeur épithéliale odontogénique calcifiée : à propos d'un cas
}

\section{The calcified epithelial odontogeneous tumor: A case report}

HABIB HAMDI*, TOUHAMI BEN ALAYA*, IMENE BEN ROMDHANE*, BASSEME KHATTECHE*, CARLE FAVRE DE THIERRENS**

\section{RÉSUMÉ}

Les tumeurs odontogéniques à composante minéralisée sont multiples. Parmi ces tumeurs, on trouve la tumeur épithéliale odontogénique calcifiée ou tumeur de Pindborg. Cette tumeur est rare. Son diagnostic est orienté par la radiologie, mais est difficile avant l'apparition des calcifications. A ce stade, le diagnostic différentiel se pose surtout avec le kyste épidermoïde ou l'améloblastome. Après l'apparition des calcifications, le diagnostic devient plus aisé, surtout si on a recours à la tomodensitométrie qui permet une excellente étude des différentes densités de la matrice tumorale. (Med Buccale Chir Buccale 2002; 8: 87-90).

mots clés : Tumeurs odontogéniques, Tumeurs calcifiées, Tomodensitométrie

\section{SUMMARY}

A case of calcifying epithelial odontogenic tumor, which is a rare neoplasm also known as Pindborg tumor, is reported. Radiographically the lesion may be unilocular multilocular, radiolucent, it must be separated clinically from the dentigerous cyst, odontogenic keratocyst, ameloblastoma and odontogenic myxoma. When a mixed radiolucentradiopaque pattern is found, clinical differential diagnosis such as adenomatoid odontogenic tumor, ameloblastic fibro-odontoma, ossifying fibroma or osteoblastoma should be considered. (Med Buccale Chir Buccale 2002; 8: 87-90).

key words : Odontogenic tumors, Radiographic diagnosis.

\footnotetext{
* Service de Médecine et Chirurgie Buccales. Faculté de Médecine Dentaire, Monastir 5000, Tunisie.

** Faculté d'Odontologie. 545 Avenue du Professeur J.L. Viala, 34193 Montpellier cedex 5, France.
} 
La tumeur épithéliale odontogénique calcifiée fait partie des multiples tumeurs odontogéniques à composante minéralisée. C'est une tumeur rare, dont le diagnostic est orienté par la radiologie. Ce cas clinique illustre l'intérêt de l'examen tomodensitométrique dans le diagnostic de cette tumeur.

\section{OBSERVATION}

La découverte par un confrère, d'une volumineuse lésion d'aspect kystique en rapport avec 41 et 42 , chez une femme de 60 ans, motive la consultation spécialisée. A l'examen clinique, on note une tuméfaction de la région symphysaire, qui fait corps avec l'os mandibulaire. 42, distoversée, est nécrosée. L'espace interdentaire entre 42 et 43 est le siège d'une poche profonde, qui fait l'objet d'une suppuration. Le cliché panoramique (Fig. 1) montre une image radioclaire étendue de 33 à 43 , avec un aspect de remaniement osseux en regard de 42,43 et 44 . Cette image radioclaire est à contour polycyclique, mal défini par endroits.

Les diagnostics cliniques et radiologiques évoqués sont un kyste péri-apical avec foyer ostéitique du coté droit en rapport avec $42,43,44$, ou un kyste épidermoide surinfecté.

L'énucléation de cette lésion; le curetage du foyer ostéitique, l'extraction de $41,42,43$ et 44 , sont pratiqués sous anesthésie générale. L'examen anatomopathologique est interprété en faveur du kyste épidermoide. L'évolution clinique immédiate est favorable.

Cependant, la patiente revient deux ans après pour une tuméfaction de la même région, avec üne poussée inflammatoire. Le cliché panoramique (Fig. 2) montre une image radioclaire symphysaire, de limites mal définies, qui s'étend médecine buccale chirurgie buccale

VOL. $8, \mathrm{~N}^{\circ} 2$ 2002 page 88
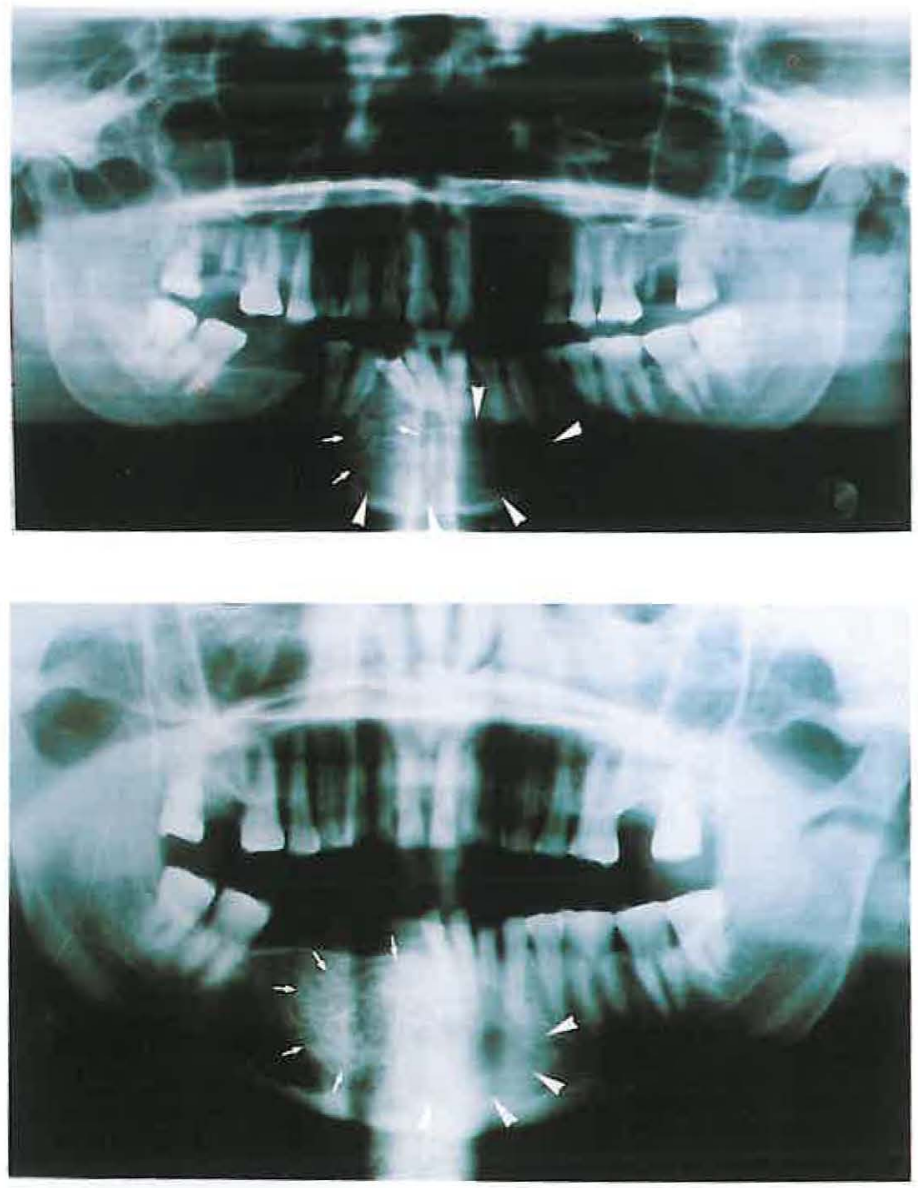

Figure 1 : Radiographie panoramique : image radioclaire symphysaire avec zone de remaniement osseux en regard de 4243-44.

Panoramic radiographic. Radiolucent lesion of the symphysis with osteosclerotic margin in the part of 42-43-44.

Figure 2 : Radiographie panoramique : image radioclaire symphysaire avec zone de remaniement osseux dense dans la région de 43-44.

Panoramic radiographic. A mixed lesion of the symphysis with radiopaque arera in the part of $43-44$. 
médecine buccale chirurgie buccale 2002

page 90

\section{COMMENTAIRES}

La tumeur épithéliale odontogénique calcifiée se traduit par un foyer ostéolytique radioclair, de contour plus ou moins précis, pouvant être monogéodique ou polycyclique. L'image est parsemée de fines ponctuations radio-opaques, de taille et de nombre variable, le plus souvent en rapport avec une dent incluse refoulée. Le kyste odontogénique calcifié est d'aspect très proche. [1-4]. Certaines images ressemblent à celles d'un améloblastome, d'un fibrome améloblastique ou d'un kyste épidermoïde, d'un kyste folliculaire, voire d'un kyste péri-apical, surtout avant l'apparition des calcifications [3,5]. Après l'apparition de ces calcifications, le diagnostic différentiel peut se poser avec un cémentome ou un ostéome.

La tomodensitométrie permet de visualiser la matrice tumorale, et de déterminer l'extension de la tumeur, ses rapports avec les structures anatomiques voisines. Au niveau osseux péri-tumoral, on note des images de condensation, de défor- mation, de refoulement. Ce sont des signes de bénignité $[6,7]$. Au niveau de la tumeur, la tomodensitométrie montre la densité de la lésion, son caractère homogène ou hétérogène, et la présence ou non de foyers de calcifications. L'imagerie permet, au besoin, d'effectuer des mesures de densités, qui peuvent donner une meilleure approche de la nature histologique de la lésion. Le recours à cet examen est justifié dés que le volume de la tumeur est important, ou reste mal défini par la radiographie standard [4,6-10].

Ce cas clinique illustre le fait que le bilan radiologique standard est souvent insuffisant. Le recours à la tomodensitométrie a mis en évidence une zone dense au sein d'une hypodensité de limite polycyclique, ce qui a permis de ré-orienter le diagnostic préalablement posé.

Ce travail montre que, malgré l'importance de l'examen clinique et histologique, l'imagerie moderne facilite l'approche diagnostique toujours complexe des images lacunaires des maxillaires [11].

\section{RÉFÉRENCES}

1 - BARTHE M, Daudibertières L. Classification radiologique des tumeurs bénignes ostéo-mandibulaires. Rev Odontostomatol 1994; 23: 57-66.

2 - Califano L, ZUPI $A_{1}$ Vetrani $A$. Tumeur épithéliale odontogénique calcifiante ou tumeur de Pindborg. Rev Stomatol Chir Maxillo Fac. 1993; 94: 110-4.

3 - Chomette G, Auriol M, Guilbert F. Tumeur épithéliale odontogéne calcifiée bifocale (tumeur de Pindborg). Rev Stomatol Chir Maxillo Fac, 1984; 85: 329-36.

4 - favre Dauvergane E, Auriol M, Le Charpentier Y. Tumeurs odontogéniques. Encycl Méd. Chir, Editions Techniques (Paris), Stomatologie-Odontologie I, 22062 F.10, 1995: 10p.

5 - ChOmette G, Auriol M. Classification des tumeurs bénignes et kystes des maxillaires. Rev Stomatol Chir Maxillo Fac, 1985; 86: 277-84.

6 - Dubayle P, Boyer B, Le Chainch P, Pajoni D. Imagerie maxillo-faciale : la tomodensitométrie. Encycl Méd Chir, Editions Techniques (Paris), StomatologieOdontologie I, 22-010 D.30, 1996: 7p.
7 - DUVERnEUIL. NM, ChIRAZ J. Imagerie maxillo-faciale. Flammarion, Paris, 1997; 90-1.

8 - Leyder P, Ruf R, Texier J, Laufer J. Tumeurs osseuses mandibulaires, les difficultés du diagnostic. Rev Stomatol Chir Maxillo Fac, 1984; 85: 320-4.

9 - PAJONI D. La radiographie panoramique, lecture, pièges, limites. Rev Odontostomatol 1992; 216: 44962.

10 - Pasquet G, Cavevian R. La tomodensitométrie en odonto-stomatologie : une approche diagnostique. Inf Dent 1982; 16: 1511-30.

11 - Torres $J H_{1}$ favre de Thierrens $\mathrm{C}$, Jacquard $\mathrm{C}$ JACQUEY $N$. Trois tumeurs bénignes agressives du maxillaire supérieur. Cah Oto-rhino-laryngol 1998; 33: 433-41. 